\title{
Aprender el sistema de escritura: Un estudio de las modalidades de intervención de los adultos en situaciones de alfabetización familiar en poblaciones urbano - marginadas de Argentina
}

\section{Learning the writing system: A study about the intervention methods by adults in family literacy situations from urban-marginalized towns in Argentina}

\author{
Alejandra Stein ${ }^{1}$ \\ Celia Renata Rosemberg ${ }^{2}$ \\ Universidad de Buenos Aires, Argentina \\ (Rec: Diciembre 2010 - Acep: Abril 2011)
}

\begin{abstract}
Resumen
Este artículo presenta un análisis de situaciones de alfabetización que tuvieron lugar en hogares de poblaciones urbano-marginadas de Argentina, a partir de la implementación de un programa de alfabetización familiar. El análisis se centra en las intervenciones de los adultos durante actividades focalizadas en el aprendizaje del sistema de escritura -juegos con sonidos y letras, establecimiento de correspondencias grafema-fonema, escritura de palabras-. Los resultados mostraron que estas actividades adoptan un formato asimétrico, similar a la secuencia iniciación-respuesta-evaluación identificada en el ámbito escolar. Los adultos producen numerosas autorreparaciones de las preguntas que formulan a los pequeños. En las actividades de escritura se observó el modelado, el dictado de palabras mencionando el nombre de las letras y la colaboración en el análisis fonológico de las palabras. Los niños tienen la oportunidad de reconocer y trazar letras, atender a los sonidos del habla, establecer correspondencias grafema-fonema y escribir sus primeras palabras.

Palabras clave: alfabetización temprana, alfabetización familiar, interacción, sistema de escritura.
\end{abstract}

\begin{abstract}
The paper reports a qualitative analysis of literacy situations generated by a family literacy program- that took place in the homes of children from poor urban population of Argentina. The analysis is centred in adult interventions during activities focused in different abilities required to use the writing system -sound games and writing activities. Results showed that the activities adopt an asymmetric format similar to the sequence initiation-response-feedback observed in the classroom. It was also observed that adults frequently autorepair the directives and questions they make to the children. In the writing activities adults and older children model, dictate the name of the letters and help the little children to analyse the sounds of the words. The children have the opportunity to recognize and write letters, pay attention to the sounds of the words and establish phoneme-grapheme correspondences.
\end{abstract}

Key words: early literacy, family literacy, interaction, writing system.

\footnotetext{
1 Correspondencia a: Alejandra Stein. Dirección Postal: Gavilán 4350, PB 3, C1419ENR, Ciudad de Buenos Aires, Argentina. Tel: 54-11-6091-9363. E-mail: alejandrastein@yahoo.com.ar.

2 Celia Renata Rosemberg. Dirección Postal: Rómulo Naón, 2783, C1430EQA, Ciudad de Buenos Aires, Argentina. Tel: 54-11-45422214. E-mail: crrosem@hotmail.com.
} 


\section{Introducción}

En las últimas décadas, el estudio del proceso de alfabetización temprana ha recibido especial atención, en tanto numerosos trabajos realizados en diferentes países (Borzone, 1997; Landry \& Smith, 2006; Neuman, 2006; Snow, Porche, Tabors \& Harris, 2007) han mostrado que los elevados índices de fracaso escolar registrados en grupos de sectores de bajo nivel socioeconómico -niños de poblaciones minoritarias y marginadas- reflejan las dificultades de estos niños para aprender a leer y escribir. Las investigaciones mencionadas pusieron asimismo de manifiesto que el éxito o las dificultades en el dominio de la lectura y la escritura en la escuela primaria están ligados a una serie de conocimientos y de habilidades que comienzan a desarrollarse a edad temprana en las interacciones que el niño mantiene con los miembros de su familia en el contexto del hogar (Borzone, 1997; Snow, 1983; Sulzby \& Teale, 1987; Taylor, 1983). En el marco de estos planteos, este estudio se centra en el análisis de las primeras experiencias de alfabetización en el hogar, en las que tienen la oportunidad de participar niños de poblaciones urbano marginadas de Argentina.

\section{Los conocimientos y las habilidades implicados en el proceso de alfabetización temprana}

Uno de los sistemas conceptuales más conocidos de los conocimientos y las habilidades implicados en el proceso de alfabetización temprana es el propuesto por Whitehurst y Lonigan (2003). Este sistema diferencia en el desarrollo de los precursores de la alfabetización dos dominios o dimensiones de información independientes durante la etapa preescolar: la dimensión denominada inside-out -de adentro hacia fuera- y la llamada outside-in -de afuera hacia adentro-. El primero se refiere a las fuentes de información que provienen de las palabras escritas y cuyo dominio requiere por parte de los niños habilidades para traducir los grafemas en fonemas y los fonemas en grafemas, es decir, la decodificación o traducción de las unidades escritas en representaciones fonológicas. Por su parte, la dimensión llamada outside-in incluye aquellas fuentes de información externas a las palabras escritas que constituyen un soporte para la comprensión del significado del texto -vocabulario, conceptos, esquemas narrativos, entre otros elementos-. La alfabetización requiere el desarrollo de habilidades para emplear estos dos dominios de información.
Otros sistemas conceptuales organizan a los componentes de la alfabetización temprana según se refieran al desarrollo de habilidades lingüísticas generales o a aspectos directamente vinculados con la escritura (Landry \& Smith, 2006; Snow, 2006). Las habilidades lingüísticas generales incluyen la comprensión y el empleo de vocabulario, la posibilidad de construir frases y oraciones gramaticalmente correctas y de utilizarlas en un discurso extendido, así como habilidades para utilizar el lenguaje de manera flexible con el fin de responder a las demandas de diferentes contextos sociales. Por su parte, los aspectos vinculados con la escritura se refieren a los conocimientos acerca de las letras, a la conciencia fonológica y a las convenciones de la escritura -como por ejemplo, la orientación de la escritura-, a sus diferencias respecto del dibujo y al reconocimiento de que la escritura transmite significados.

Borzone, Rosemberg, Diuk, Silvestri y Plana (2004) identifican las mismas habilidades en el proceso de alfabetización, pero las integran en un marco conceptual diferente. Conciben el proceso de alfabetización en términos amplios, como el desarrollo integral de los cuatro usos del lenguaje -hablar y escuchar, leer y escribir-. De modo independiente de la modalidad -oral o escrita-, el proceso de alfabetización implica el dominio progresivo de la posibilitad de recrear el mundo a través del lenguaje. El proceso conlleva la adquisición progresiva de una serie de conocimientos y habilidades que responden a tres órdenes diferentes: los niños tienen que aprender que la escritura es lenguaje, tienen que llegar a dominar el sistema de escritura y tienen también que adquirir el estilo discursivo particular que caracteriza a la escritura y que la diferencia del estilo de discurso propio de las interacciones orales "cara a cara" (Borzone, 1997). El estilo de discurso escrito, implica el uso de vocabulario preciso, una sintaxis compleja, de formas lingüísticas descontextualizadas en la elaboración de distintos tipos de textos: explicación, descripción, narración, entre otras.

Aún cuando todos los trabajos mencionados reconocen la importancia del desarrollo de habilidades lingüísticas generales en el proceso de alfabetización, porque todas estas habilidades serán luego empleadas en la producción de textos escritos, el acceso y el uso de la escritura requiere necesariamente el conocimiento y el dominio de los componentes del sistema de escritura. 


\section{¿Qué habilidades y conocimientos conlleva el dominio del sistema de escritura?}

El dominio del sistema de escritura involucra conocimientos referidos a la forma específica en que cada sistema de escritura representa el lenguaje -unidades linguiísticas representadas por las grafías- y a las convenciones de orientación. Estos conocimientos y habilidades, que están implicados en los procesos de transcripción y decodificación (Berninger \& Swanson, 1994; Borzone \& Diuk, 2001; Sánchez Abchi, 2008), constituyen procesos básicos o de nivel inferior que pueden incidir en las habilidades de nivel superior -de composición y de comprensión-, tanto en el proceso de adquisición de la escritura y de la lectura como en etapas posteriores de su desarrollo.

Diversos estudios sobre el aprendizaje del sistema de escritura, pusieron de manifiesto que los niños escriben en formas preconvencionales antes de lograr escribir en forma convencional (Braslavsky, 1992). Los primeros trabajos realizados en esta línea se centraron en la descripción de estas primeras formas de escritura y en su desarrollo, en relación con las características de linealidad y diferenciación de rasgos de la escritura convencional (Borzone, 1997). En esta línea, Lavine (1972) señaló que a la edad de 3 años la mayoría de los niños producen formas que contienen características propias de la escritura y no del dibujo -linealidad y orientación horizontal-. Los estudios descriptivos de la forma externa de la escritura de niños entre 3 y 6 años de edad pusieron de manifiesto una progresión en los trazos realizados por los pequeños desde el garabato no organizado, líneas en zigzag sin variación en la forma, hasta escrituras más diferenciadas con ordenamiento lineal de los elementos y formas marcadas y aproximaciones a las letras o letras convencionales (Legrün, 1932; Hildreth, 1936; ambos citados en Gibson \& Levin, 1975).

Los primeros intentos de los niños por leer y escribir evidencian sus esfuerzos por dominar el sistema de escritura. Los niños tienen que descubrir qué unidades del lenguaje -sonidos, sílabas o palabras- representan las letras. Los sistemas de escritura alfabéticos como el español representan la estructura fonológica de las palabras. Es por ello que para poder leer y escribir en este tipo de sistema es necesario tomar conciencia de que las palabras están formadas por sonidos e inducir las correspondencias grafema-fonema (Borzone, 2003).

Read mostró que las escrituras tempranas dan cuenta de los conocimientos de los niños acerca de la escritura y del sistema de escritura (Read, 1971). Read observó que estas escrituras inventadas se basan en un principio fonético y que las correspondencias grafema-fonema se derivan del conocimiento del nombre de las letras. De este modo, los niños demuestran tener conocimientos referidos a la estructura fonológica de las palabras, al principio alfabético y a los patrones convencionales de representación de la ortografía.

Sin embargo, comprender las relaciones entre las grafías y los sonidos no resulta una tarea sencilla porque a los niños les cuesta percatarse de que los sonidos conforman las palabras (Ehri \& Roberts, 2006). Escuchan una palabra que conocen e inmediatamente activan su significado atravesando los sonidos, como si la forma sonora de la palabra no existiera. El lenguaje es para los niños como un vidrio (Luria, 1980). Los niños tienen dificultad en descubrir que las palabras están formadas por sonidos porque al pronunciarlas, los sonidos se articulan juntos. Como no los pueden distinguir en su propia articulación, entonces, no pueden establecer las relaciones entre éstos y las letras (Borzone, 1997). La habilidad para atender a los sonidos de las palabras se denomina conciencia fonológica (Borzone \& Signorini, 2002).

El desarrollo de las habilidades de conciencia fonológica en niños hablantes de distintas lenguas ha sido abordado en estudios correlacionales, longitudinales, predictivos, experimentales y con intervención, en los que se ha atendido a la incidencia de distintas variables en el desarrollo (Wagner \& Torgensen, 1987; Brady \& Shankweiler, 1991; Defior, 1994; Borzone, 1997; Signorini, 1998). Algunos trabajos han considerado el papel de la conciencia fonológica como factor causal de la habilidad lectora (Wagner \& Torgensen, 1987; Defior 1994; Phillips \& Torgesen, 2006); otros han mostrado que ciertas habilidades de conciencia fonológica se desarrollan como consecuencia de la enseñanza de escritura alfabética (Morais, 1991). Sin embargo, diversos trabajos (Morais, Cary, Alegria \& Bertelson, 1979; Liberman, Liberman, Mathingly \& Shankweiler, 1980; Perfetti, 1992) mostraron que existe una relación recíproca entre la conciencia fonológica y el aprendizaje de la lectura y la escritura: la conciencia fonológica facilita el aprendizaje de la lectura y la escritura y ésta, a su vez, promueve un mayor desarrollo de la conciencia fonológica.

Estos distintos tipos de relaciones entre la conciencia fonológica y el aprendizaje de la lectura y la escritura son atribuibles al hecho de que la 
conciencia fonológica constituye un fenómeno componencial o multifactor (Signorini, 1998). En este sentido, los trabajos de Burgess (2006) y Phillips y Torgesen (2006) han puesto de manifiesto la existencia de diferentes niveles de conciencia fonológica, esto es, diferentes grados de conocimiento explícito de la estructura fonológica de las palabras (Manrique \& Gramigna, 1984; Yopp, 1988; Jiménez, 1992; Signorini \& Borzone, 1996). La conciencia fonológica no constituye un fenómeno unitario, sino multifactor, formado por un conjunto de habilidades de dificultad creciente $y$ de distinto orden de emergencia en el desarrollo. En una escala de dificultad creciente, pueden distinguirse el reconocimiento y la producción de rimas, la identificación de sonidos, la segmentación fonológica, el conocimiento de las correspondencias grafema-fonema y la recodificación fonológica. Las habilidades fonológicas que preceden al aprendizaje de la lectura y la escritura, son la sensibilidad hacia las rimas y la sílaba. En cambio, otras habilidades de mayor complejidad -las habilidades que implican manipular fonemas, tales como la elisión o la inversión- parecen requerir de una exposición sistemática a la escritura (Perfetti, 1991).

Algunas investigaciones han revelado diferencias en las habilidades fonológicas de niños de diferente origen social (Borzone, 1997; Borzone y Diuk, 2001). Estas diferencias sociales pueden atribuirse a diferencias en las intervenciones de los adultos que interactúan con los niños en las situaciones en las que está presente la escritura. De hecho, algunos trabajos experimentales mostraron el impacto de estrategias específicas de intervención para promover el desarrollo de estas habilidades (Bradley \& Bryant, 1983; Lundberg, Frots \& Petersen, 1988) y proporcionan evidencias empíricas que sugieren la importancia del apoyo y la intervención de un adulto competente para el logro de estos aprendizajes por parte de los niños.

Por su parte, los estudios realizados en el contexto del hogar han identificado los tipos de actividades de lectura y escritura que se llevaban a cabo en las familias (Taylor, 1983; Taylor \& Dorsey Gaines, 1988; Leseman \& Van Tuijl, 2006; Reyes, Alexandra \& Azuara, 2007), así como diferencias en la frecuencia en la que los niños de sectores medios o trabajadores participaban en actividades en las que se hacía un uso educativo o recreativo de la lectura y la escritura, tales como la lectura compartida de un cuento o la escritura de una carta frente al niño (Taylor, 1983; Taylor \& Dorsey Gaines, 1988; Leseman \& Van Tuijl, 2006; Reyes et al., 2007).
Empero, en estos estudios no se analizaron detalladamente las modalidades de intervención de los adultos durante la consecución de estas actividades, que pueden facilitar en los niños el acceso a los conocimientos y las habilidades implicadas en el dominio del sistema de escritura; dominio que progresivamente permitirá a los niños el uso del sistema como una herramienta cultural para transmitir y comunicar información. El objetivo del presente trabajo ${ }^{3}$ es precisamente analizar en situaciones de alfabetización familiar generadas a partir de la implementación de un programa de desarrollo lingüístico y cognitivo infantil, en hogares de poblaciones urbano marginadas de Argentina ${ }^{4}$, las modalidades de intervención de los adultos que en actividades de escritura y de juegos con sonidos pueden promover las oportunidades que los niños tienen de aprendizaje de la escritura.

\section{Método}

\section{Corpus}

El corpus está conformado por 300 actividades que tuvieron lugar en 124 situaciones de alfabetización familiar generadas a partir de la implementación de un programa de desarrollo lingüístico y cognitivo infantil en los hogares de 30 niños de 4 años de barrios urbano marginados de la ciudad de Córdoba, la ciudad de Buenos Aires y el Gran Buenos Aires, Argentina ${ }^{5}$. Estas actividades se focalizaron en diversos conocimientos y habilidades necesarios para el dominio del sistema de escritura. Se incluyen 84 actividades de escritura -a) escritura del nombre (45); b) escritura de otras palabras (32),

\footnotetext{
Este trabajo forma parte de una investigación más amplia: "El entorno de alfabetización en hogares de niños preescolares de sectores urbano-marginados. Un estudio de los procesos interaccionales en las situaciones de lectura y escritura". Tesis doctoral (2010) de Stein. A.

4 El programa "Oscarcito. Promoción y desarrollo lingüístico y cognitivo de niños pequeños en contextos de pobreza" (C. R. Rosemberg y A. M. Borzone) se elaboró como transferencia de resultados del proyecto "El desarrollo lingüístico y cognitivo en la primera infancia. Un estudio psicolingüístico y sociocultural en barrios urbano marginales de Buenos Aires" (CONICET, PIP 5591; SECyT, PICT BID 1201 OC- AR No4-14153, A. M. Borzone, directora); con el aporte de las fundaciones Care de Alemania y Árcor de Argentina.

5 Los niños y sus familias viven en barrios urbano marginales -poblaciones denominadas habitualmente "villas de emergencia"- de Buenos Aires y de Córdoba, Argentina. Se trata de inmigrantes o descendientes de inmigrantes del norte de Argentina y de países limítrofes (Bolivia, Paraguay y Perú). Dada la situación de pobreza en la que viven, no cuentan con materiales de lectura y escritura.
} 
escritura de frases (7)- y 216 actividades focalizadas en el desarrollo de habilidades fonológicas.

El programa de desarrollo lingüístico y cognitivo infantil involucra la consecución de doce talleres de formación para las familias, en los que se abordan diversos aspectos del desarrollo lingüístico y cognitivo infantil -aprendizaje de palabras, habilidades fonológicas, reconocimiento y trazado de letras, habilidades discursivas, entre otros- y los modos en los que los adultos pueden promoverlos en el hogar. Dichos talleres se llevaron a cabo en centros comunitarios y jardines de infantes. En cada uno de los talleres se les entrega a las familias un libro infantil. Se trata de libros interculturales (serie "En la Casa de Oscarcito") especialmente diseñados, que recuperan la vida cotidiana de los niños de estas poblaciones y, al mismo tiempo, les permiten el acceso a otras formas e instrumentos culturales. En el contexto de lo que les sucede a los personajes del libro, se presentan juegos y actividades destinadas a promover el acceso a la alfabetización.

Los libros incluyen los siguientes tipos de actividades: lectura y renarración de cuentos, desarrollo de vocabulario y conceptos -por ejemplo, adivinanzas-, juegos con instrucciones -lotería, memotest, recorrido, dominó, entre otros- y diversas actividades focalizadas en el desarrollo de los conocimientos y habilidades implicados en el sistema de escritura. Estas últimas comprenden: actividades de trazado tales como recorridos y laberintos; escritura de palabras -nombre propio y otras palabras- y actividades focalizadas en el desarrollo de habilidades fonológicas -recitado de versos, reconocimiento e invención de rimas, identificación de sílaba inicial (por ejemplo, aparear dos palabras que comienzan con la misma sílaba),identificación de sonido inicial (por ejemplo, colorear los dibujos de las palabras que comienzan con sonido inicial /a/), establecimiento de correspondencias entre fonemas y grafemas (por ejemplo, identificación de fonema inicial omitido en una palabra, completar la palabra escrita con el grafema correspondiente; aparear vocales con imágenes de palabras que comienzan con el sonido correspondiente). El corpus que se analiza en el presente trabajo comprende específicamente las actividades implicadas directamente en el acceso al sistema de escritura.

Las familias que participan del programa están conformadas por adultos con un bajo nivel de escolaridad y por niños mayores que asisten a la escuela primaria o secundaria; se identificó sólo el caso de un hermano mayor que asiste a la universidad. Los adultos, quienes alcanzaron en promedio 7 años de escolaridad, han logrado un dominio básico o escaso de la lectura y la escritura y no están familiarizados con la lectura de cuentos. Debido a ello, los adultos, en muchos casos, se apoyan en los niños mayores que se encuentran escolarizados para la consecución de la situación de lectura.

\section{Obtención de información empírica}

La recolección de datos se llevó a cabo mediante observaciones audiograbadas -registro de la interacción verbal- que fueron complementadas con registros escritos acerca de otros rasgos (interacciones kinésicas, gestuales, disposición espacial de los participantes, entre otros) de cada una de las situaciones de alfabetización familiar en las que participaron los niños.

Para lograr una aproximación ecológica a las situaciones en estudio, en el presente trabajo, aunque se les proporcionó a las familias los libros de cuentos y de actividades y se les brindó información acerca del desarrollo lingüístico infantil en los talleres, no se pautó externamente quiénes debían participar de las situaciones de alfabetización junto con el pequeño ni ningún otro aspecto contextual de las situaciones.

\section{Análisis de la información}

Se realizó un análisis cualitativo de cada una de las actividades de alfabetización empleando el Método Comparativo Constante (Glaser \& Strauss, 1967; Strauss \& Corbin, 1991), con el fin de generar categorías que dieran cuenta de: a) la estructura de la situación; b) las intervenciones que los adultos y los niños mayores ponían en juego en su interacción con los pequeños para lograr la consecución de las actividades y c) los conocimientos y las habilidades que los niños tenían la oportunidad de aprender en las situaciones. Posteriormente, se cuantificaron algunas de las dimensiones analizadas con el objetivo de conocer su distribución en el corpus analizado.

\section{Resultados}

En términos generales, puede decirse que el conjunto de actividades analizadas configuran situaciones asimétricas, reguladas por el adulto y que cuentan con una escasa participación espontánea de los pequeños. El adulto es quien, proporcionando información y mediante directivas -más o menos explícitas- y preguntas que focalizan la atención del niño, guía al pequeño en la realización de la actividad. El niño, por su parte, participa siguiendo las indicaciones de su interlocutor. 
Gráfico 1. Tipos de intervenciones de los adultos y de los niños mayores en las actividades focalizadas en habilidades fonológicas y en el establecimiento de correspondencias grafema - fonema.

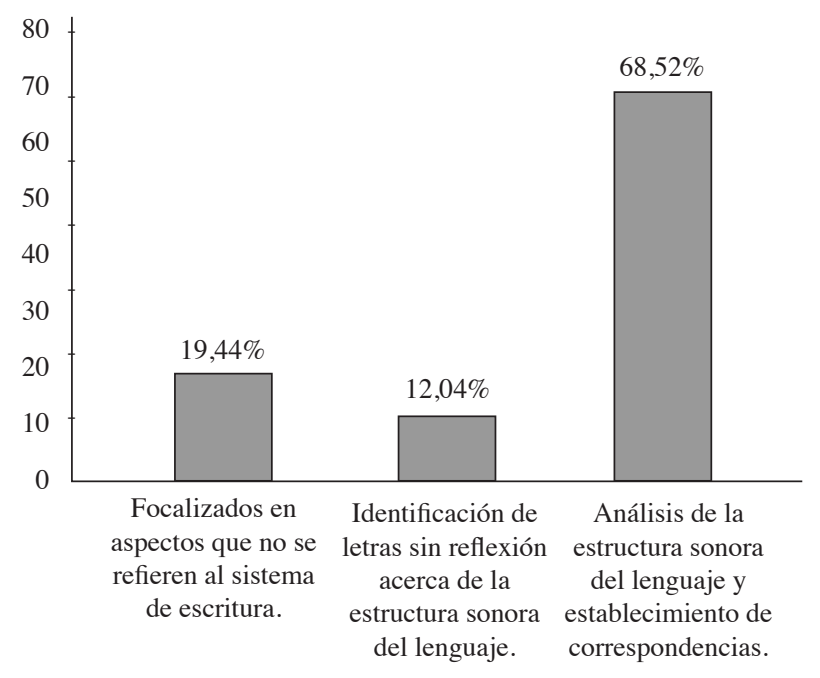

Durante la consecución de estas actividades los niños realizan escasas iniciaciones, se limitan a responder a las demandas e indicaciones del adulto o solicitan un mayor apoyo de su interlocutor. En efecto, mientras escriben, los pequeños piden explícita y activamente indicaciones y sostén para la tarea -preguntan cómo es, cómo se escribe, cómo sigue-, buscan la confirmación de sus acciones -formulan preguntas tales como ¿está bien?, ¿así?, ¿acá?-, buscan la palabra escrita para usarla como modelo y le muestran a otra persona lo que escribieron.

Estas actividades se caracterizan por el aparente interés del adulto o niño mayor en alcanzar la respuesta o resolución correcta de la actividad y por las abundantes autorreparaciones (Sacks, Schegloff \& Jefferson, 1977; Schegloff, 1979) que éstos realizan de las directivas y preguntas que formulan a los pequeños. En efecto, los adultos suelen comenzar planteando una pregunta amplia -por ejemplo, "De estos seis dibujos, ¿cuál empieza con a?”- y luego, ante las dificultades de los pequeños para responder, van restringiendo las posibles respuestas. Cuando los niños se equivocan, los adultos evalúan negativamente sus emisiones, repiten la pregunta, proveen la respuesta correcta, repiten la palabra prolongando el sonido inicial, señalan la letra nombrada por el niño para marcar el error, entre otras formas de intervención, tal como se observa en el intercambio que se presenta a continuación, entre Alexander y su madre ${ }^{6}$.

6 Para la presentación de los fragmentos de intercambio que ilustran las diversas categorías de análisis presentadas en los resultados, se emplearon las siguientes codificaciones de
(1) \{Alexander realiza con su madre una actividad que tiene por finalidad identificar el sonido inicial de una serie de objetos ilustrados\}.

Madre: Pintá de rojo las cosas que empiezan con lal, de verde las cosas con lel y de amarillo las cosas que empiezan con /u/. De estos 6 dibujos, ¿cuál empieza con /a/?

Alexander: /a/ de elefante.

Madre: No, elefante no tiene /al. Fijate, desde acá empezá. ¿Cuál empieza con /a/? Alexander: ((señala el dibujo de una uña)). Madre: Este es uña. ¿Este qué es? Uno ((señala una imagen con el número 1$)) . ¿ Y Y$ este es una...?

Alexander: Escoba.

Madre: ¿Empieza con /a/?

Alexander: No.

Madre: ¿Y este qué es?

Alexander: Un avión.

Madre: ¿Cuál empieza con /a/?

Alexander: \{contento\} Un avión.

Jefferson (2004):

(silencio) pausa larga interturno

:vocal prolongada

::: incremento en la prolongación de la vocal

$((\mathrm{XXX}))$ acción no verbal

$\{\mathrm{XX}\}$ comentarios del observador / transcriptor ¿X? Entonación ascendente para interrogaciones ¡X! Entonación ascendente para exclamaciones se-pa-ra-ción separación en sílabas

Por último, las letras fueron transcriptas entre $<>$, los fonemas entre / / y el texto leído de manera literal se colocó entre "'. 
En el intercambio, mediante una secuencia de preguntas, respuestas y evaluaciones, la madre de Alexander focaliza la atención del niño en la identificación de los sonidos iniciales de las palabras - ¿Empieza con /a/?-, le proporciona información -Este es uña-y realiza correcciones -No, elefante no tiene /a/- cuando el pequeño se equivoca. Las intervenciones de la madre focalizan la atención del niño en los dibujos cuyos nombres no empiezan con /a/ -uña, elefante, escoba-. Luego de señalarle que ninguno de ellos empieza con /a/, conduce finalmente al niño a atender al dibujo de "avión", fomentando, de ese modo, el proceso de comparación y discriminación del pequeño.

\section{Análisis de las actividades focalizadas en el desarrollo de habilidades fonológicas y en el establecimiento de correspondencias grafema-fonema}

Las actividades destinadas a promover el desarrollo de habilidades fonológicas de los niños comprenden el recitado y la repetición de versos y trabalenguas, actividades de producción de rimas y de reconocimiento de sílaba inicial, de sonido inicial o de fonema omitido y el establecimiento de correspondencias grafema-fonema.

Tal como se observa en el Gráfico 1 que se presenta a continuación, en el conjunto de estas actividades se identificaron distintos tipos de intervenciones por parte de los adultos y de los niños mayores.

Como se aprecia en el gráfico, durante la consecución de estas actividades se identificaron intervenciones focalizadas en aspectos que no se refieren al sistema de escritura $(19,44 \%)$; intervenciones que requieren que los niños nombren las letras y los dibujos, pero en las que no se reflexiona acerca de los sonidos del habla $(12,04 \%)$; y, por último, intervenciones focalizadas en el análisis de la estructura sonora del lenguaje y el establecimiento de correspondencias grafema-fonema $(68,52 \%)$.

La mayor parte de los hogares $(68,52 \%)$ se caracterizan porque los adultos y los niños mayores intervienen para guiar la atención del niño hacia los sonidos del habla y para promover el establecimiento de correspondencias entre los grafemas y los fonemas. Así por ejemplo, en las actividades de recitado de versos y trabalenguas, se observaron situaciones en las que los adultos les proponen a los pequeños recitar el verso a otra persona conocida o, como sucede en el ejemplo que se presenta a continuación, el adulto o el hermano mayor lee el verso, lo repite y luego lo recita por partes de manera tal que el niño lo repita junto con él, focalizando la atención del pequeño en la estructura sonora de las palabras.

\section{(2) \{Brenda, de 13 años, le lee un verso a su hermana Patricia\}.}

Hermana: A ver, repetí este trabalenguas con

Oscarcito. Vos repetí cuando yo diga "En la casa de Mariana”.

Patricia: En la casa de Mariana.

Hermana: "Se escondieron bananas".

Patricia: Se escondieron mananas [bananas].

Hermana: "El domingo a la mañana".

Patricia: El domingo a la mañana.

Si bien las situaciones de recitado y repetición de versos constituyen una oportunidad para que los niños comiencen a atender a los sonidos del lenguaje, solo en dos situaciones se observó que los adultos se refieren explícitamente a la inclusión de rimas en el verso recitado, como se observa en el siguiente fragmento de intercambio entre Brisa y su madre.

\section{(3) \{La madre de Brisa lee un verso\}.}

Madre: $\{$ Lee $\}$ Hola, hola, nos encontramos otra vez. Te digo un versito que inventé: Yo soy Oscarcito, el que salta como un sapito". ¿Viste como rima? Yo soy Oscarcito, el que salta como un sapito.

\section{Brisa: \{Repite con su mamá\} isapito!}

Los niños pequeños, por su parte, repiten el verso o trabalenguas junto con el adulto cuando éste lo solicita -de lo contrario, escuchan en silencio- y en los casos en los que el verso es breve, lo dicen solos, sin el apoyo de sus interlocutores.

Otras actividades destinadas a promover el desarrollo de habilidades de conciencia fonológica conllevan por parte de los niños la identificación de la sílaba inicial de las palabras apareando palabras que comienzan igual. En estas actividades se observó que los adultos y los niños mayores que interactúan con los pequeños, suelen nombrar dos palabras de la serie y les preguntan a los pequeños si estas palabras "suenan igual". También se observó que para facilitar la inferencia del niño, enfatizan la pronunciación de la sílaba inicial de las palabras nombradas. Asimismo, durante la consecución de la actividad, los adultos y los niños mayores restringen las opciones de respuesta y reformulan la consigna, produciendo autorreparaciones, cuando se pone de 
manifiesto que ni la consigna ni las intervenciones iniciales del adulto son suficientes para que el niño comprenda la tarea que debe realizar. En algunos casos, en la reformulación de la consigna, los adultos llaman explícitamente la atención de los niños en torno a los sonidos de las palabras, prolongándolos.

En tanto, aquellas actividades que tienen por objeto el reconocimiento del sonido inicial y el establecimiento de correspondencias entre los sonidos y las letras iniciales de las palabras, implican generalmente aparear una serie de dibujos con el grafema que representa el fonema inicial. En estas actividades, las intervenciones de los adultos elicitan los nombres de los dibujos y de las letras. El etiquetamiento de los dibujos da lugar, en algunos casos, a focalizarse en el vocabulario, tanto en relación a la estructura sonora de las palabras como en relación a los conceptos y significados subyacentes, tal como se observa en el intercambio que se presenta a continuación.

(4) \{Jonathan y su madre nombran los dibujos de la actividad\}.

Madre: Este dibujo ¿qué es? ((señala el dibujo)).

Jonathan: Un nono.

Madre: ¿Qué vuela por el aire?

Jonathan: ((no contesta)).

Madre: ¿Qué vuela?

Jonathan: Un avome [avión].

Madre: ¡Eso! ¿Qué dijiste? A ver.

Jonathan: Un avón \{más fuerte\}.

Madre: Avión.

Jonathan: Avón.

Madre: ¿Qué vocal es?

Jonathan: $<e>$.

Madre: No.

Jonathan: $<o>$.

Madre: Si dijiste a:::vión.

Jonathan: Avón.

Madre: ((se sonríe)). ¿Qué dijiste hijo? Avión.

Jonathan: Avón.

Madre: ¿Qué vocal es?

Jonathan: $<a>$.

Madre: $<a>$.

Como se observa en el fragmento presentado, la madre de Jonathan intenta que el niño nombre el objeto avión, definiéndolo por su función específica. Cuando el niño nombra el objeto -avome, avón-, la madre reestructura fonológicamente la emisión del niño, brindándole la forma adulta -avión-. Luego, conduce al niño a atender al sonido inicial. Al prolongar este sonido en el marco de la matriz de intercambio, crea un contexto en el que el niño puede más fácilmente identificar el sonido y establecer las correspondencias con las grafías.

En estas actividades se observó también que cuando los niños tienen que reconocer letras, las intervenciones de los adultos conllevan distintas demandas para los niños. Así por ejemplo, mientras algunos adultos formulan preguntas referidas a la identificación de los nombres de las letras a los niños, otros las nombran ellos mismos y luego solicitan que los niños las repitan. Además, en algunos casos -como el de la madre de Brisa-, prolongan el fonema representado por la letra nombrada.

(5) Madre: Bueno, vamo[s] a poner la letra e::., la o:::, i::: ((acomoda una serie de fichas de cartón con letras)).

Brisa: La $<o>$. Yo pono [pongo].

Madre: ((Señalando)). ¿Qué letra es esa?

Brisa: La i::.. Esta va ahí. ((Acomoda las

fichas con las letras sobre la mesa)). Así, así, así.

Madre: a::..

Brisa: a::.

Madre: e::.:

Brisa: e::.. No, vamo[s] 'peza' [empezar] de пиеvo.

Madre: Vamo[s] a empezar de nиеvo. A.:.

Brisa: a:::.

Madre: e::.:

Brisa: $i: \ldots$.

Madre: o:.:.

Brisa: o::. U:::

Madre: Muy bien.

Luego de nombrar los dibujos y las letras, los adultos colaboran con los niños para que establezcan correspondencias entre fonemas y grafemas. En algunos casos, el adulto informa, establece las correspondencias y se las comunica al niño. En otras situaciones, solicitan por medio de preguntas que los niños sean quienes establezcan las correspondencias - ¿Con qué letra empieza?, ¿Con qué vocal va?-y proveen apoyo a través de diferentes movimientos interaccionales, tales como prolongar los sonidos que representan las letras y el sonido inicial de la palabra; mostrar la forma en que se ponen los labios para pronunciar el sonido correspondiente 
a la letra y ocultar la palabra con la mano dejando a la vista únicamente la primera letra. Asimismo, señalan cada uno de los dibujos que es necesario aparear con las letras y preguntan si empieza con el sonido inicial planteado en la consigna; también dicen otras palabras que comienzan con el sonido indicado en la consigna.

En el siguiente intercambio, Alexander nombra los dibujos y su madre proporciona información acerca de las correspondencias grafema - fonema y prolonga el sonido inicial de las palabras.

(6) Madre: Ahora seguí todos los puntitos y dibujá la $<a>$.

Alexander: $(($ Escribe la $<a>))$.

Madre: Con la $<a>$ se inicia a:::vión \{prolonga el sonido inicial\}. ¿Qué es esto? ((señala el dibujo de un avión)).

Alexander: Avión.

Madre: la/ de a:::vión \{prolonga el sonido inicial\}.

En las actividades en las que además de reconocer el sonido inicial los niños deben completar con la letra correspondiente, el adulto lee la palabra sin el sonido inicial y pregunta "¿con qué hay que completar?”, "¿qué le falta para que diga...?” y dice la palabra completa. En el fragmento de intercambio entre Candela y su madre que se presenta a continuación, se ilustra el andamiaje del adulto en la consecución de esta actividad.

(7) Madre: Bueno, escuchame, acá dice \{lee\}

"Oscarcito se olvidó algunas letras, ayudalo

a completar estas palabras". Escuchame, mirá, acá dice "jo" y ¿qué es lo que es esto? ((Señala el dibujo del ojo)) o::. ¿Qué es?

Candela: Ojo.

Madre: ¿Y qué le falta ahí para que diga o:::jo?

Candela: /a/.

Madre: Ajo va a decir. Si es un o::.jo.

Candela: jo.

Madre: Ahí dice "jo". ¿Qué le faltaría para que diga o:::jo?

Candela: No sé.

Madre: ¿Cómo que no sabés? Si ahí... la letra $<o>$.

Candela: $\langle o\rangle$.

Madre: Ponemos la $<o>$ adelante. ¿A ver?
Respecto del desempeño de los niños, se observó que, en muchas de las situaciones observadas, los pequeños cometen errores al nombrar las letras -confunden letras con números o con colores-, al identificar el sonido inicial de las palabras y al establecer las correspondencias grafema-fonema. $\mathrm{Al}$ equivocarse, suelen probar distintas respuestas hasta alcanzar la correcta. Asimismo, repiten la respuesta correcta dada por el adulto, se muestran inseguros y solicitan su evaluación al responder. En algunos casos, no tienen un desempeño parejo a lo largo de una misma actividad: son inestables, responden de manera correcta e incorrecta alternativamente.

En los casos en los que hay un mejor desempeño de los niños, éstos nombran letras y dibujos correctamente y logran establecer las correspondencias entre grafemas y fonemas. El siguiente intercambio entre Brisa y su madre ilustra este tipo de situación.

(8) Madre: ¿Iglú con qué letra empieza de las vocales? Allá, $i::$ glú, ¿con qué letra?

Brisa: Con la $<i>$.

Madre: A ver si nos acordamos cuál era $l a<i>$.

Brisa: ((Golpea en la mesa el lugar donde se encuentra la vocal)).

Madre: Esa, bueno. A ver, esto es...((señala el dibujo de un racimo de uvas)).

Brisa: Una uva.

Madre: Ajá, ¿con quéletra va? ¿Adónde va?

Brisa: $u::$.

Madre: Con la u:.:. La de uva. Vamo[s] a poner la uvita ahí.

Como se muestra en el gráfico 1, un pequeño porcentaje $(19,44 \%)$ de las intervenciones de los adultos y de los niños mayores se centraban en aspectos que no se referían al sistema de escritura. Así por ejemplo, se observaron situaciones de recitado de versos y trabalenguas en las que tanto los movimientos interaccionales del adulto como los del niño se centran en el contenido del texto y no en la identificación o repetición de rimas, sin atender a los sonidos del habla.

(9) \{La madre de Jonathan lee un verso\}. Madre: Mira qué bonito. \{Lee\} "Teresa poné la mesa y si no hay pan poné la cabeza. Poné tu cabeza". ¿Quién es Teresa?

Jonathan: ¿A qué? ¿A qué pasa?

Madre: No, aquí estamos. ¿Quién es Teresa? Mira, es bonito, Jonathan. Mira hijo, esto te vuelvo a leer 


\section{(...).}

Madre: "Teresa poné la mesa y si no hay pan poné tu cabeza". ¿Dónde pone su cabeza Teresa?

Jonathan: De su pelo.

Madre: No.

Jonathan: Es una nena.

Madre: Viste que no entendiste.

Jonathan: Pan.

Madre: Mira, Teresa poné la mesa y si no hay pan poné tu cabeza.

Jonathan: Tu cabeza.

Madre: Encima de...

Jonathan: Comila [comida].

Madre: No, mesa.

Jonathan: Mesa.

Madre: Como no hay pan, entonces pone la cabeza.

Jonathan: Y puedo comer.

En el ejemplo se pone de manifiesto que ni el niño ni su madre parecen estar familiarizados con el formato de recitado y repetición de versos. Asimismo, en el intercambio que se presenta a continuación, en el que participan Alexander y su madre, se ponen de manifiesto tanto las dificultades de los niños para producir rimas, como las de los adultos para colaborar con ellos.

(10) Madre: Escuchá estas. \{Lee\} "Son las ocho, comete un bizcocho". A ver, vos inventá una, a ver. Son las...

Alexander: ¿Bizcochos?
Madre: No, pero acá ya te dieron una idea, mirá. Son las ocho, comete un bizcocho. Decime otra. ¿Qué hora?

Alexander: Mi hora del relo [reloj] tengo que buscar.

Madre: El reloj no, mi amor. Yo te estoy preguntando si acá te dan una idea que son las ocho que te comas un bizcocho, a ver vos qué podés pensar.

Alexander: Ya sé, coman.

Madre: Sí, pero tenés que decir una hora y decir qué comen.

Alexander: Qué comen una hora.

Madre: A ver, son las nueve, comete una nieve. A ver escribí.

Alexander: Pero grande.

Madre: Son las... Acá te vamos a poner ((escribe en una hoja)) son las nueve... nueve \{prolonga todos los sonidos\}. Son las nueve, comete una...

Alexander: Una nube.

Madre: Nieve.

Como se observa en el ejemplo, la directiva de la madre -A ver, vos inventá una, a ver. Son las...- no es suficiente para que el niño comprenda que debe producir un verso cuya última palabra termine con los mismos sonidos que tiene la última palabra del verso anterior. Posteriormente, la madre evalúa negativamente la respuesta del niño y formula una autorreparación de su pregunta inicial, en la que reitera el verso que se proporciona como ejemplo en el libro, en un aparente intento de incitar al niño a producir un verso con ese modelo -No, pero acá ya te dieron una idea, mirá. Son las ocho, comete un

Gráfico 2. Intervenciones de los adultos y de los niños mayores en las actividades de escritura.

(Los niños escriben con el apoyo de un adulto o de un niño mayor)

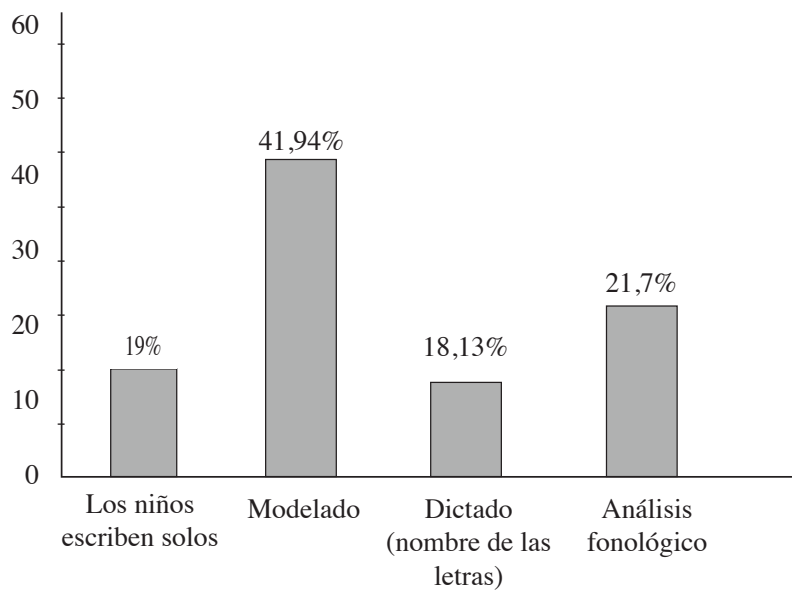


bizcocho. Decime otra. ¿Qué hora? -. Sin embargo, esta nueva intervención de la madre, al focalizarse en el contenido semántico -Decime otra. ¿Qué hora?-y no mostrar el paralelismo sonoro, no es suficiente para lograr que el niño comprenda que tiene que producir dos versos que terminen con los mismos sonidos. Las intervenciones siguientes de la madre, como continúan focalizándose en el contenido semántico -tenés que decir una hora y decir qué comen-, tampoco son suficientes para reparar la fractura de la comunicación con su hijo y lograr que éste comprenda lo que se espera que haga. Finalmente, es la madre quien produce el verso que rima -son las nueve, comete una nieve-. Pero el niño sigue sin comprender el objetivo de la actividad, tal como se pone de manifiesto en la última intervención del pequeño que completa el verso de la madre con la palabra "nube".

En las actividades centradas en la identificación del sonido inicial de los nombres de objetos ilustrados y en el establecimiento de correspondencias grafema-fonema en las que la consigna consiste en establecer relaciones entre una serie de ilustraciones y las letras que representan el sonido inicial de sus nombres, se observó también que, en ocasiones, las intervenciones de los adultos y de los niños mayores no se focalizan en aspectos referidos al sistema de escritura. En estos casos, los adultos se limitan a formular preguntas referidas a los nombres de los dibujos o leen la consigna de la actividad incluida en los materiales de alfabetización, pero preguntan sólo por los nombres de las ilustraciones que comienzan con el fonema indicado y no reflexionan acerca del sonido inicial de las palabras junto con el niño. En el siguiente intercambio entre Denis y su madre, en la consecución de la actividad, sólo nombran los objetos ilustrados.

(11) Denis: Ete [este] e[s] ave... aión [avión] ((señala el dibujo)).

Madre: Araña ((señala el dibujo)).

Denis: Aaña [araña].

Madre: Ojo, ¿no? lel de estrella, escoba ((señala los dibujos)).

Denis: Ecoba [escoba], etrella [estrella].

Madre: Oído, ¿no?

Denis: Oio [oído], ¿no?

\{Continúan nombrando los dibujos\}

Otro pequeño número de las intervenciones de los adultos y de los niños mayores $(12,04 \%$, ver gráfico 1) se centran en la identificación y denominación de letras -aspectos del sistema de escritura-, pero no focalizan la atención de los niños en los sonidos que conforman las palabras. Así por ejemplo, en el intercambio que se presenta a continuación, Jonathan, su madre y Joseline, la hermana de 12 años del pequeño, señalan las palabras escritas, pero no pronuncian las palabras de manera tal que el niño atienda a los sonidos y pueda identificar aquellas palabras que comienzan con la misma sílaba. De este modo, aún cuando la actividad puede permitir el reconocimiento por parte del niño de la letra inicial, las intervenciones no configuran una matriz que conduzca al niño a atender a los sonidos, generando oportunidades para el desarrollo de habilidades de conciencia fonológica.

(12) Madre: $\{$ Lee\} "Uní los dibujos de las palabras que empiezan igual”. Media...

Hermana: Con mesa.

Jonathan: ((señala un dibujo)).

Madre: Media con...

Jonathan: Con este por eso (( señala un dibujo equivocado)).

Madre: No.

Hermana: Sí. Media, con la eme $\{<m>\}$. Jonathan: No.

Madre: ¿Cuál está aquí con la letra eme $\{<m>\}$ ? A ver, fijate, fijate acá, ¿cuál es con la letra eme $\{<m>\}$ ? Con este mira, con esta letra (( señala la primera letra de la palabra "media")).

Hermana: Con la eme $\{<m>\}$.

Madre: Igualito a este, fijate, a ver...

Jonathan: (( señala la letra $<m>$ en “mesa")).

Madre: Muy bien, ese uní con la media.

De modo similar, en diversas actividades que tienen por objeto el reconocimiento del sonido inicial y el establecimiento de correspondencias grafema-fonema, los niños sólo nombran y colorean letras o resuelven la actividad junto con un adulto o hermano mayor, sin reflexionar acerca de la estructura sonora del lenguaje.

\section{Análisis de las actividades de escritura}

En las actividades de escritura del nombre propio, de otras palabras y de frases breves, se observó que en la mayoría de los casos (81\%) los niños escriben con el apoyo de un adulto o de otro niño mayor. Sólo en el 19\% de las situaciones de escritura del propio nombre los niños escriben de modo autónomo, en las restantes situaciones de escritura 
del nombre y en el conjunto de las actividades de escritura de otras palabras o frases, los niños reciben el apoyo de sus interlocutores para escribir.

En el gráfico 2 se presentan los porcentajes de las situaciones en las que los niños escriben solos y en las que escriben con el apoyo de las intervenciones de los adultos y de los niños mayores, así como también el porcentaje de los distintos tipos de intervenciones que éstos emplean para andamiar el desempeño infantil.

Como se observa en el gráfico, dentro de las actividades de escritura en las que los niños escriben con la ayuda de un adulto o de un niño mayor, se identificaron casos en los que los adultos escriben un modelo para que el niño copie $(41,94 \%)$, situaciones en las que dictan los nombres de las letras $(18,13 \%)$ y situaciones en las que los adultos estimulan a los niños a realizar un análisis fonológico de las palabras y a establecer correspondencias entre las grafías y los sonidos del habla $(21,7 \%)$. En 4 casos se observaron indicios de que los niños comienzan a realizar, durante la actividad de escritura, un análisis fonológico de las palabras.

En el intercambio que se presenta a continuación, como sucede en el $41,94 \%$ de los casos, Patricia escribe su nombre copiando el modelo escrito por su madre.

(13) Madre: \{lee\} “Yo soy...” ¿Cómo te llamás vos?

Patricia: Patri.

Madre: Bueno, ponga Patri. ¿Querés que te copie? ¿Me pasás una lapicera?

((Toma una lapicera y comienza a escribir $)$ ).

Madre: Patri \{Lee en voz alta lo que escribió\}. Acá arriba Patricia ((señala dónde debe escribir la niña)).

Patricia: ((copia el nombre escrito por su madre en silencio)). Ya está.

En otros casos $(18,13 \%)$, los adultos colaboran con los niños diciéndoles los nombres de las letras que deben escribir, como se observa en el intercambio que se presenta a continuación entre Alexander y su madre.

(14) \{Alexander escribe "oreja" con la ayuda de su madre\}.

Madre: Aquí mi amor ((señala el lugar donde Alexander debe escribir)). $L a<O>$, la ere $\{<r\rangle\}$.

Alexander: ((Escribe las letras $<o>y<r>))$.

Madre: $L a<e>$.
Alexander: $(($ Escribe la letra $<e>))$.

Madre: La jota $\{<j>\}$.

Alexander: ((Escribe la letra “ $j ”))$.

Madre: Y la $<a>$.

Alexander: ((Escribe la letra $<a>))$.

Madre: O:::rrre:::jjja::: \{pronuncia la palabra prolongando los sonidos\}. ¿Viste?

Alexander: Lo hice.

Por último, en otros casos en los que los niños escriben con ayuda, se observó que las intervenciones de los adultos y de los niños mayores se focalizan en aspectos fonológicos $(21,7 \%)$, como se muestra en el siguiente intercambio en el que Candela escribe la palabra "cresta" con el apoyo de su madre.

(15) Candela: Cres-ta \{pronuncia la palabra separándola en sílabas\}.

Madre: Cre:sss-ta: \{repite la emisión de la niña, prolongando algunos sonidos\}.

Candela: ta \{repite la sílaba final de la palabra\}.

Madre: La de Candela ponemos acá, acá ((señala el lugar donde Candela debe escribir)).

Candela: cacaca \{intenta pronunciar el sonido inicial de la palabra\}.

Madre: Ahí arriba ((nuevamente, le indica dónde debe escribir)).

Candela: ((Escribe la letra $<c>))$.

Madre: La ce $\{<c\rangle\}$ de Candela. La rrrrr

$\{$ prolonga el sonido\} de Roberto.

Candela: ((Escribe la letra $<r>))$.

((Candela continúa escribiendo con la ayuda de su madre)).

En el intercambio, la madre de Candela prolonga los sonidos correspondientes a las letras que se deben escribir y dice palabras conocidas que comienzan con ese sonido. La niña, por su parte, intenta realizar un análisis fonológico de la palabra que está escribiendo, prolongando el sonido inicial -cacaca- y separándola en sílabas -cres-ta-.

En las interacciones registradas, las intervenciones de los niños muestran, en algunos casos, evidencia de que los pequeños han comenzado a desarrollar habilidades de conciencia fonológica, tal como se muestra en el siguiente intercambio entre Alexander y su madre.

(16) \{La madre de Alexander ayuda a su hijo a escribir la palabra "gato"\}. 
APRENDER El SISTEMA DE ESCRITURA: Un ESTUDiO DE LAS MODALIDADES DE INTERVENCIÓN DE LOS ADULTOS EN SITUACIONES DE ALFABETIZACIÓN FAMILIAR EN POBLACIONES URBANO-MARGINADAS DE ARGENTINA

Madre: Detrás de esta pared, ¿qué animal estará escondido?

Alexander: Un gato.

Madre: Muy bien. Tenés que escribir el nombre del gato. Ga-to \{separa la palabra en sílabas\}.

Alexander: Esperá ((da vuelta la página y se fija en el ejercicio anterior en el que está escrita la palabra gato)). ;Ay, ay! ((tapa la palabra)). Ya me lo acordé.

Madre: A ver aquí ((señala dónde debe escribir)).

Alexander: ((comienza a escribir)).

Madre: Ya está la primera letra. ¿Qué sigue?

Alexander: ((escribe)).

Madre: Bueno, lo empezás a escribir de пиеvо. Ga...

Alexander: to to ((escribe la $<t>))$.

Madre: toooo \{prolonga la $<o>$ \}.

Como se observa en el intercambio, Alexander, para escribir, se autodicta la palabra, repitiendo la sílaba final. El hecho de que el niño se autodicte la palabra al escribirla, repitiendo la última sílaba y prolongando el sonido final puede constituir una evidencia de que ha comenzado a recurrir a una estrategia analítica, esto es, a realizar un análisis fonológico de la palabra.

El análisis de estas actividades puso asimismo de manifiesto que en ellas los niños tienen también la oportunidad de adquirir otros conocimientos referidos al sistema de escritura tales como las convenciones de la escritura -espacios entre palabras y aspectos ortográficos- $y$ de aprender conocimientos y habilidades referidas a la escritura como modalidad de lenguaje -en particular, funciones de la escritura-. Al mismo tiempo, aprenden a regular el tamaño de las grafías, la importancia de la prolijidad $\mathrm{y}$ aspectos instrumentales, por ejemplo, el manejo del lápiz, como se observa en el siguiente intercambio entre Candela y su madre.

(17) \{Candela escribe un verso ("Candela, la que alumbra como una vela") con el apoyo de su madre\}.

Candela: ((Escribe)).

Madre: Ahora dejamos un espacio. Dibujá el palito.

Candela: ((Escribe la letra $<l>))$.

Madre: La $<a>$ de Ana.

Candela: $(($ Escribe la letra $<a>))$.
Madre: Hacé bien esa $<a>$. Bueno, pero no apretés tanto $\{$ se refiere a la punta del lápiz sobre la hoja, mientras Candela traza la letra\}. A...... llll \{dicta prolongando el sonido de las letras\}.

Candela: ((Escribe la letra $<l>))$ La a:::.

Madre: Dale, la de Luciano. Alllll.

Candela: Ala.

Por otro lado, se observó un conjunto de intervenciones de los adultos y de los niños mayores focalizadas en aspectos referidos al reconocimiento y el trazado de letras. Dentro de estas intervenciones se identificaron movimientos interaccionales que consisten en el modelado: el adulto escribe la letra o la palabra completa y se la muestra como modelo al niño, para que éste la copie. En algunos casos, además, el adulto describe la forma de la letra y toma la mano del niño para ayudarlo a escribir. El siguiente intercambio en el que Patricia escribe con la ayuda de sus hermanas, ilustra algunas de estas intervenciones.

(18) \{Patricia escribe su nombre con la ayuda de sus hermanas Brenda (13 años), Mirta (10 años) y Aylén (5 años)\}.

Mirta: Dale, escribí tu nombre. Hacé la pe $\{\langle p\rangle\}$. La pe $\{\langle p\rangle\}$, la $<o>$. La pe $\{\langle p\rangle\}$. Esta es la pe $\{<p>\}$, mirá ((escribe la letra y se la muestra a su hermana)). Esta es la pe $\{<p>\}$.

Patricia: ((escribe)).

(...).

Brenda: Ahora la te $\{<t>\}$.

Patricia: ¿La te $\{<t>\}$ ?

Brenda: Dos palitos.

Patricia: ((escribe)).

Brenda: La erre $\{\langle r\rangle\}$. La $<i>$, un palito.

Aylén: Como de indio.

(...).

Brenda: Patri mirá, dame tu mano. Así, de ahí para arriba y para abajo ((toma la mano de Patricia y la mueve)).

En todas las interacciones registradas se observaron numerosas intervenciones de los adultos y de los niños mayores que evalúan positivamente el desempeño de los pequeños. Estas intervenciones que resaltan los progresos en el desempeño de los niños pueden predisponer positivamente al pequeño en la actividad concreta y contribuir a su imagen como escritor. 


\section{Discusión}

Los resultados del presente estudio revisten particular relevancia en tanto muestran en detalle las características particulares que adoptan las situaciones de juegos con sonidos y escritura en los hogares de niños que crecen en sectores urbanos marginados.

El análisis de estas actividades mostró que en ellas los niños tienen la oportunidad de aprender conocimientos y habilidades referidos al sistema de escritura, en particular, el reconocimiento y el trazado de letras, la atención a los sonidos del habla, el establecimiento de correspondencias entre grafemas y fonemas, así como diversos aspectos instrumentales de la escritura. Las investigaciones previas realizadas en el contexto internacional y en diferentes lenguas mostraron la importancia del desarrollo de todas estas habilidades como precursores del proceso de alfabetización (Borzone, 1997; Borzone, 2003; Goswami, 2003; Burgess, 2006; Ehri \& Roberts, 2006; Lonigan, 2006; Phillips \& Torgesen, 2006).

A diferencia de los estudios previos que identificaron y enumeraron los tipos de actividades de lectura y escritura que se llevaban a cabo en las familias (Taylor, 1983; Taylor \& Dorsey Gaines, 1988; Leseman \& Van Tuijl, 2006; Reyes et al., 2007), en esta investigación el análisis se focalizó en la descripción detallada de las modalidades de intervención de los adultos y de los niños mayores, en tanto la calidad de estas intervenciones -el aspecto en el que se focalizan, así como el modo en que lo hacen- afecta las oportunidades de aprendizaje de los niños. En este sentido, los datos analizados pusieron de manifiesto que tanto aquellas actividades destinadas al desarrollo de habilidades fonológicas, al establecimiento de correspondencias entre grafemas y fonemas y a la escritura de palabras y frases adoptan, a diferencia de las actividades de lectura y narración de cuentos analizadas en otro estudio (Stein \& Rosemberg, 2010) ${ }^{7}$, un formato similar al que con frecuencia se observa en el ámbito escolar (Sinclair \& Coulthard, 1975): una secuencia de iniciaciones por parte del adulto, respuestas del niño y evaluaciones del adulto. El análisis, asimismo, mostró que para lograr la consecución de la actividad, los adultos y los niños mayores deben producir con frecuencia autorreparaciones (Sacks et al., 1977; Rosemberg \& Silva, 2009) de las directivas y de

\footnotetext{
Stein, A. y Rosemberg, C. R. (2010). "Compartir cuentos en el hogar. Diferentes estilos de lectura en poblaciones urbano marginadas de Argentina”. En preparación.
}

las preguntas que formulan a los pequeños. Ello puede atribuirse a la dificultad que implican estas actividades para el niño que se enfrenta por primera vez a la escritura, no domina el contenido y tampoco está habituado al particular formato interaccional.

Las autorreparaciones del adulto o del niño mayor de sus intervenciones representan, en el contexto de estas actividades de enseñanza, ajustes progresivos que, a la manera de "un andamiaje", permiten que el niño pequeño pueda desempeñarse en su "zona de desarrollo próximo" (Vygotsky, 1978) y construir nuevos conocimientos. Las dificultades que implica la consecución de estas actividades para los participantes, podrían verse también incrementadas por el hecho de que los adultos y los hermanos mayores tienen un nivel de alfabetización relativamente bajo. Aunque de hecho pueden hacer un uso procedural del sistema -leer y escribir textos simples-, pueden no poseer conocimientos declarativos del sistema y habilidades metalingüísticas que les permitan, por ejemplo, deslindar los elementos del lenguaje oral -fonemas, sílabas- y establecer relaciones explícitas entre ellos y con los elementos del lenguaje escrito. De este modo, puede resultarles difícil colaborar con los niños para que focalicen la atención en los distintos elementos, puedan identificarlos y relacionarlos. De ahí que se observen en el corpus de datos numerosas autorreparaciones que los adultos producen de sus propias emisiones para resolver situaciones de incomprensión, ajustar de modo continuo sus intervenciones y facilitar la tarea a los niños, cuando los pequeños no pueden responder a la actividad.

En las actividades de escritura, los adultos y los niños mayores andamian el desempeño del pequeño por medio de intervenciones similares a aquellas identificadas por Snow (1983) en hogares de nivel socioeconómico medio. Aunque en el presente trabajo se observó que predomina el modelado, también se identificó el dictado de palabras mencionando el nombre de las letras y la colaboración en el análisis fonológico de las palabras.

El análisis pormenorizado de las interacciones mostró que en un tercio de los casos, en las actividades focalizadas en el desarrollo de habilidades fonológicas, los adultos en realidad no se centran en aspectos que involucran la reflexión sobre la estructura fonológica de las palabras. En estos casos, la resolución de la actividad no se alcanza por medio de un andamiaje del adulto o del niño mayor que interactúa con el pequeño, sino por medio de pistas semánticas u ortográficas proporcionadas por el adulto que conducen a la respuesta correcta, pero 
no involucran por parte del niño pequeño el proceso cognitivo esperado a partir del objetivo de la tarea.

En este sentido, constituyen estrategias de contextualización que no pueden ser consideradas un andamiaje (Rosemberg, 2002; Manrique, 2008). Aún cuando estas diferencias en las intervenciones de los adultos y de los niños mayores pueden, en parte, atribuirse al nivel de desarrollo real de los niños, es necesario tener en cuenta que ellas implican distintas demandas y oportunidades de aprendizaje para los niños.

La trascendencia de estas intervenciones de los adultos y de los niños mayores identificadas en gran parte de las actividades analizadas, radica en el hecho comprobado en diversas investigaciones (Goswami, 2003) de que algunas de las habilidades que forman parte de la conciencia fonológica no se desarrollan automáticamente con la edad, sino que requieren de la instrucción directa y de los juegos de reconocimiento y producción de rimas, sílabas y sonido inicial de las palabras (Bradley \& Bryant, 1983; Lundberg y otros, 1988; Ehri, 2000). Del mismo modo, el conocimiento de las letras y la inferencia de las correspondencias entre las letras y los fonemas que permite el uso progresivo de la escritura de palabras y textos depende de la inclusión de los niños en formatos de interacción con adultos, en el marco de actividades tales como las que se analizan en este trabajo.

\section{Referencias}

Borzone, A. M. (1997). El proceso de alfabetización en niños pequeños: diferencias socioculturales. Tesis doctoral no publicada. Universidad de Buenos Aires. Buenos Aires, Argentina.

Borzone, A. M. (2003). Leer y escribir a los 5. Buenos Aires: Aique.

Borzone, A. M.; Rosemberg, C.; Diuk, B.; Silvestri, A. \& Plana, D. (2004). Niños y maestros por el camino de la alfabetización. Buenos Aires: Red de Apoyo Escolar.

Borzone, A. M. \& Diuk, B. (2001). El aprendizaje de la escritura en español: Estudio comparativo entre niños de distinta procedencia social. Interdisciplinaria, 18, 1-33.

Borzone, A. M. y Signorini, A. (2002). El aprendizaje inicial de la lectura. Incidencia de las habilidades fonológicas, de la estructura de la lengua, de la consistencia de la ortografía y del método de enseñanza. Linguiística en el Aula, 5, 29-48.

Bradley, L. \& Bryant, P. (1983). Categorizing sound and learning to read - a causal connection. Nature, 301, 419-421.

Brady, S. \& Shankweiler, D. (1991). Phonological Processes in Literacy. Hillsdale NJ: Lawrence Erlbaum.

Braslavsky, B. (1992). La escuela puede. Una perspectiva didáctica. Buenos Aires: Aique.

Braslavsky, B. (2005). Enseñar a entender lo que se lee. Buenos Aires: Fondo de Cultura Económica.

Burgess, S. (2006). The development of phonological sensitivity. En D. K. Dickinson \& S. B. Neuman (eds.) Handbook of
Early Literacy Research, Vol. 2 (pp. 90-100). New York: The Guilford Press.

Defior, S. (1994). La conciencia fonológica y la adquisición de la lectoescritura. Infancia y Aprendizaje, 67-68, 91-113.

Ehri, L. C. (2000). Learning to read and learning to spell: Two sides of a coin. Topics on language disorders, 20 (3), 19-36.

Ehri, L. C. \& Roberts, T. (2006). The roots of learning to read and write: acquisition of letters and phonemic awareness. En D. K. Dickinson \& S. B. Neuman (eds.), Handbook of Early Literacy Research, Vol. 2 (pp. 113-131). New York: The Guilford Press.

Gibson, E. J. \& Levin, H. (1975). The Psychology of Reading. Massachusetts: The MIT Press.

Glaser, B. \& Strauss, A. (1967). The discovery of grounded theory. Chicago: Aldine Publishing Company.

Goswami, U. (2003). Early phonological development and the acquisition of literacy. En D. K. Dickinson \& S. B. Neuman (eds.), Handbook of Early Literacy Research (pp. 111-125). New York: Guilford Press.

Hildreth, G. (1936). Developmental sequences in name writing. Child Development, 7, 291-303. Citado en Gibson, E. J. \& Levin, H. (1975). The Psychology of Reading. Massachusetts: The MIT Press.

Jiménez, J. E. (1992). Metaconocimiento fonológico: Estudio descriptivo sobre una muestra de niños prelectores en edad preescolar. Infancia y Aprendizaje, 57, 49-66.

Landry, S. H. \& Smith, K. E. (2006). The influence of parenting on emergent literacy skills. En D. K. Dickinson \& S. B. Neuman (ed.), Handbook of Early Literacy Research, Volume 2 (pp. 135-148). New York: Guilford Publications, Inc.

Lavine, L. O. (1972). The development of perception of writing in pre-reading children: A cross-cultural study. Tesis doctoral no publicada, Departamento de Humanidades de Cornell University. Citado en Gibson, E. J. \& Levin, H. (1975). The Psychology of Reading. Massachusetts: The MIT Press.

Legrün, A. (1932). Wie und was "schreiben" Kindergartenzöglinge? Zeitschrift für pädagogische Psychologie. Citado en Gibson, E. J. \& Levin, H. (1975). The Psychology of Reading. Massachusetts: The MIT Press.

Leseman, P. P. M. \& van Tuijl, C. (2006). Cultural Diversity in early literacy: findings in Dutch Studies. En D. K. Dickinson \& S. B. Neuman (ed.) Handbook of Early Literacy Research, Vol. 2 (pp. 211-228). New York: Guilford Publications, Inc.

Liberman, I., Liberman, A., Mathingly, I. \& Shankweiler, D. (1980). Orthographic and the beginning reader. En J. Kavanagh \& R. Venezky, (eds.), Orthography, reading and dyslexia (pp. 137-153). Baltimore: University Park Press.

Lundberg, I., Frots, J. \& Petersen, O. (1988). Effects of an extensive program for stimulating phonological awareness in preschool children. Reading Research Quarterly, 23, 263-284.

Luria, A. (1980). Conciencia y lenguaje. Madrid: Pablo del Río Editor.

Manrique, M. S. (2008). Conversación y desarrollo lingüístico en la infancia. Un estudio de la interacción verbal en el jardín de infantes con población urbano-marginal. Tesis doctoral no publicada. Universidad de Buenos Aires. Buenos Aires, Argentina.

Manrique, A. M. \& Gramigna, S. (1984). La segmentación fonológica y silábica en niños de preescolar y primer grado. Lectura y vida, 5, 4-13.

Morais, J. (1991). Constraints on the development of phonemic awareness. En S. Brady \& D. Shankweiler (eds.), Phonological processes in literacy. Hillsdale, NJ: Erlbaum.

Morais, J.; Cary, L.; Alegria, J. \& Bertelson, P. (1979). Does awareness of speech as sequence of phones arise spontaneously? Cognition, 7, 323-331. 
Perfetti, Ch. (1991). Representations and awareness in the acquisition of reading competence. En L. Rieben \& Ch. Perfetti (eds.), Learning to read. Basic research and its implications (pp. 33-44). NJ: Lawrence Erlbaum.

Perfetti, Ch. (1992). The representation problem in reading acquisition. En P. B. Gough, L. C. Ehri \& R. Trieman (eds.), Reading acquisition (pp. 107-143). NJ: Erlbaum.

Phillips, B. M. \& Torgesen, J. K. (2006). Phonemic awareness and reading: beyond the growth. En D. K. Dickinson \& S. B. Neuman (eds.), Handbook of Early Literacy Research, Vol. 2 (pp. 101-112). New York: The Guilford Press.

Reyes, A., Alexandra, D. \& Azuara, P. (2007). Las prácticas de lectoescritura en los hogares de inmigrantes mexicanos. Infancia y aprendizaje, 19 (4), 395-407.

Rosemberg, C. R. (2002). La conversación en el aula: aportes para una teoría de la enseñanza a través del discurso. Tesis doctoral no publicada. Universidad de Buenos Aires. Buenos Aires, Argentina.

Rosemberg, C. R. \& Silva, M. L. (2009). Teacher - children interaction and concept development in kindergarten. Discourse processes, 46, (6) 572-591.

Sacks, H. E., Schegloff, A. \& Jefferson, G. (1977). A simplest systematic for the organization of turn-taking for conversation. Language, 50, 696-735.

Share, D. L. \& Stanovich, K. E. (1995). Cognitive processes in early reading development: Accomodating individual differences into a model of acquisition. Issues in Education, 1, 1-57.

Signorini, A. (1998). La conciencia fonológica y la lectura. Teoría e investigación acerca de una relación compleja. Lectura y Vida, XIX, 3, 15-22.

Signorini, A. \& Borzone, A. M. (1996). Las habilidades metafonológicas, la lectura y la escritura en niños de cinco años. Lenguas Modernas, 23, 71-93.
Sinclair, J. M. \& Coulthard, R. M. (1975). Towards an analysis of discourse. The English used by teachers and pupils. Oxford: Oxford University Press.

Snow, C. E. (1983). Literacy and language: relationships during the preschool years. Harvard Educational Review, 53 (2), 165-189.

Snow, C. E. (2006). What counts as literacy in early childhood? En K. McCartney \& D. Phillips (eds.), Handbook of early child development (pp. 274-294). Oxford: Blackwell.

Strauss, A. \& Corbin, J. (1991). Basics of cualitative research. Grounded theory. Procedures and technics. Londres: Sage Publications.

Sulzby, E. (1989). Assessment of writing and of children's language while writing. En L. Morrow \& J. Smith (eds.), The role of assessment and measurement in early literacy instruction. Englewoods Cliffs, NJ: Prentice Hall, 83-109.

Taylor, D. (1983). Family Literacy. En Exeter. NH; Heineman. Taylor D. \& Dorsey Gaines, C. (1988). Growing up literate: Learning from the inner city families. Portsmouth, NJ: Ablex.

Vygotsky, L. (1978). El desarrollo de los procesos psicológicos superiores. México: Grijalbo.

Wagner, M. M. \& Torgesen, J. K. (1987). The natural of phonological processing and its causal role in the acquisition of reading skills. Psychological Bulletin, 101, 192-212.

Whitehurst, G. J. \& Lonigan, C. (2003). Emergent Literacy: Development from prereaders to readers. En S. B. Neuman \& D. K. Dickinson (eds.), Handbook of Early Literacy Research (pp. 11-29). New York: the Guilford Press.

Yopp, H. K. (1988). The validity and reliability of phonemic awareness tests. Reading Research Quarterly, 23, 159-177. 